\title{
Statement from the international research interdisciplinary school collaboration group
}

Biomedical journals have become aware of the increased need for receiving manuscripts of higher quality in respect of research design and questions investigated. During the experience of conducting IRIS'es since 2006, the number of journals that recognized the International Research Interdisciplinary School (IRIS) as a promising way to address this issue has grown and now includes 10 journals.

By publishing this statement, the journals joining the IRIS collaboration group accept its goals and values, which are as follows:

- to sponsor and promote the IRIS research practicum - to support the IRIS training in designing methodologically sound research projects provided to young researches and their mentors, especially in countries in which teaching research methodology and academic writing is not part of university school curricula

- to consider these IRIS -trained young researchers and their mentors as their future authors who will be submitting manuscripts of high quality for publication

- to consider these IRIS young researchers their future reviewers and thus improve the quality of peer review process

- to create a platform for exchange of ideas and opinions between editors on their common interest

By joining the IRIS collaboration group, journals may increase their international visibility and be recognized for their contribution to increasing the quality of research performed.

\section{RESEARCH SCHOOL DESCRIPTION:}

The Research School provides an interdisciplinary international environment for training skills required for preparing research study protocols, scientific manuscript and international collaboration. During four days, the participants experience a process of elaborating and presenting common research project. This process includes defining a research topic of common interest, selecting an adequate study design, selecting an adequate method of data collection, analysis and interpretation, and developing a feasible study plan and timeline for the project, including the preparation of a research paper.

\section{ELIGIBLE PARTICIPANTS AND METHODS}

Undergraduate and postgraduate biomedical students and researchers from biomedical research in medicine, physiology, biophysics, engineering, computer science, etc. The participation is open also for mentors who would like to share the experience of this learning method.

The course consists of four workshops and is based on the Research Practicum of the Duke University, Durham, NC, USA. It combines plenary and small group discussions, project development and class presentation, and discussion on publishing scientific papers with representatives of medical scientific journals. Up to 20-25 participants work in groups to ensure an intensive interaction between participants and faculty. There is approximately equal number of local and foreign participants.

RESEARCH SCHOOL GOALS:

The goals for this research school are to:

- Develop skills to carry out research projects, based on a practical, problembased approach,

- Increase communication skills, including the skills in argumentation, negotiation and critical appraisal in English language

- Develop skills in international research team building and networking, and establish enjoyable cross discipline/cross cultural collaboration,

- Encourage researchers to publish scientific papers.

Values:

- international collaboration and networking

- interdisciplinary approach to research

- high level of professionalism in research

In addition, the journals accept to:

- publish the announcement of the forthcoming IRIS events

- $\quad$ publish the summaries on recent IRIS events

- request for manuscript review by IRIS participants

- optionally organize and sponsor IRIS events in their countries

- $\quad$ send participants and faculty to IRIS event

Editor-in-Chief: Gulmira Kudaiberdieva

Heart-Vessels and Transplantation 